\title{
The role of definitive chemoradiotherapy versus surgery as initial treatments for potentially resectable esophageal carcinoma
}

\author{
Ming-Wei Ma ${ }^{1}$, Xian-Shu Gao ${ }^{1 *}$, Xiao-Bin Gu${ }^{1}$, Mu Xie ${ }^{1}$, Ming Cui ${ }^{1}$, Min Zhang ${ }^{1}$, Ling Liu' ${ }^{1}$, Huan Yin ${ }^{2}$ \\ and Long-Qi Chen ${ }^{3 *}$
}

\begin{abstract}
Background: We performed a meta-analysis to compare the efficacy of definitive chemoradiotherapy (dCRT) and esophagectomy as initial treatments for potentially resectable esophageal cancer.

Methods: To assess both strategies, the combined odds ratios (ORs) and $95 \%$ confidence intervals (Cls) were calculated. Thirteen studies ( $N=2071$; dCRT $=869$ and surgery $=1202$ ) were included. In all, $90.39 \%$ of the patients were diagnosed with esophageal squamous cell carcinoma (ESCC).

Results: The 2-year $(\mathrm{OR}=1.199,95 \% \mathrm{Cl} 0.922-1.560 ; P=0.177)$ and 5 -year overall survival $(\mathrm{OS})$ rates $(\mathrm{OR}=0.947$, $95 \% \mathrm{Cl} 0.628-1.429 ; P=0.796)$ were not significantly different. No significant differences were identified in the 2-year OS among patients with stage I disease $(\mathrm{OR}=1.397,95 \% \mathrm{Cl} 0.740-2.638 ; P=0.303)$ or stage II- III $(\mathrm{OR}=0.418,95 \% \mathrm{Cl}$ $0.022-7.833 ; P=0.560$ ). Patients with lymph node metastases tended to have a better 5 -year OS when treated with $\mathrm{dCRT}$ than with surgery $(\mathrm{OR}=0.226,95 \% \mathrm{Cl} 0.044-1.169 ; P=0.076)$; however, the difference between the two methods was not significant. Western patients who received dCRT had poorer prognoses than patients who underwent surgery $(\mathrm{OR}=1.522,95 \% \mathrm{Cl} 1.035-2.238 ; P=0.033) . \mathrm{dCRT}$ and surgery led to similar 5-year progression-free survival rates $(\mathrm{OR}=1.06,95 \% \mathrm{Cl} 0.79-1.42 ; P=0.70)$.
\end{abstract}

Conclusions: $d C R T$ and surgery are equally effective as initial treatments for potentially resectable esophageal cancer. These results apply primarily to Asian populations as they have an increased incidence of ESCC.

Keywords: Esophageal cancer, Definitive chemoradiotherapy, Esophagostomy, Survival, Meta-analysis

\section{Background}

Among all malignancies, esophageal cancer is the sixth most common cause of cancer-related death [1]. Esophageal squamous cell carcinoma (ESCC) is the dominant type of esophageal cancer in Asia [2]. While preoperative chemoradiotherapy can improve survival and local control $[3,4]$, surgery increases the risk of comorbidities and mortality, and patients who undergo surgery may

\footnotetext{
* Correspondence: doctorgaoxs@126.com; drchenlq@scu.edu.cn ${ }^{1}$ Department of Radiation Oncology, Peking University First Hospital, No.7 Xishiku Street, Beijing 100034, People's Republic of China

${ }^{3}$ Department of Thoracic Surgery, West China School of Medicine/West China Hospital of Sichuan University, No. 37 Guoxue Alley, Chengdu 610041, Sichuan, People's Republic of China

Full list of author information is available at the end of the article
}

experience a poor quality of life [5-8]. It has been reported that even in high-volume centres, surgery alone may lead to a $5 \%$ surgical mortality rate and a $10 \%$ mortality rate overall [9]. Furthermore, older patients are at a greater risk for surgical mortality following esophagectomy [10], and the safety and therapeutic effect of preoperative chemoradiation cannot be guaranteed in centres with little experience.

In clinical practice, surgery alone is frequently used as the primary treatment modality for esophageal cancer treatment modality, especially for less advanced esophageal tumours in patients in Asian countries [1]. One study showed that the rate of pathological complete response after chemoradiotherapy was $29 \%$ for all patients 
and was as high as $49 \%$ for ESCC patients [4]. Definitive chemoradiotherapy (dCRT) is used as the initial treatment in selected patients to avoid surgical mortality [11]. In patients with persistent or recurrent disease, salvage esophagectomy may be performed. Additionally, for stage I esophageal cancer patients in Japan, studies using chemoradiotherapy have demonstrated high rates of complete response and high survival rates with mild toxicity [12]. However, data on the comparative efficacies of dCRT and surgery are insufficient.

We therefore performed a meta-analysis to compare the therapeutic effects of dCRT and esophagectomy as initial treatments for resectable esophageal cancer. Subgroup analyses based on tumour stage, lymph node metastasis, and ethnicity were also conducted.

\section{Methods}

\section{Search strategy}

This study was conducted according to the Preferred Reporting Items for Systematic Reviews and Meta-Analyses (PRISMA) guidelines [13]. Two reviewers performed an independent systematic literature search. Databases were searched for studies as follows: PubMed (1985 to May 2016) and Web of Science (1992 to June 2018). The following search terms were used: (esophageal cancer or esophageal neoplasms) and (chemoradiotherapy or chemoradiotherapy) and (esophagectomy OR surgery).

\section{Inclusion and exclusion criteria}

Studies were included if (1) they were randomised clinical trials (RCTs) or non-randomised clinical trials (nRCTs) that compared dCRT with surgery as the primary treatment in patients with resectable esophageal carcinoma, (2) they reported data on overall survival (OS) and progression-free survival (PFS) or if this information could be extracted from survival curves, and (3) the language of publication was English or Chinese. Studies that recruited patients who received neoadjuvant chemotherapy were excluded. Articles in which non-standardised scoring systems were used and those that reported insufficient data were also excluded.

\section{Data extraction}

Each study was evaluated and classified by two independent investigators. Discrepancies were resolved by discussion and/or a third reviewer. The following data were extracted and listed: first author, year of publication, demographic characteristics, treatment regimen, OS, and PFS.

\section{Data analysis}

This meta-analysis was conducted using STATA software version 12 (StataCorp, College Station, TX, USA).
The primary endpoint was OS. We assessed and quantified statistical heterogeneity using Cochran's $C$ statistic and the $I^{2}$ statistic. If heterogeneity was detected $\left(I^{2}<\right.$ $50 \%$ and $P>0.10$ ), a fixed-effects model was adopted; otherwise, a random-effects model was used. A pooled analysis was performed with the combined odds ratio (OR) and 95\% confidence intervals (CIs) using the $Z$-test. To assess potential publication bias, Begg's test and Egger's test were performed using STATA version 12. Data were considered statistically significant when $P<0.05$.

\section{Results}

\section{Characteristics of the studies}

The characteristics of the patient populations from all eligible studies are listed in Table 1. The selection process for eligible studies is shown in Fig. 1; we identified a total of 13 studies conducted between 1985 and 2015 that included 2071 patients and that compared dCRT $(N=869)$ with surgery $(N=1202)$. Of these 13 studies, $2[14,15]$ were randomised trials. The sample sizes ranged from 49 to 299 patients. Nine studies were restricted to patients with ESCC only, while 4 [16-19] enrolled patients with both ESCC and patients with adenocarcinoma; the predominant tumour histology of these 4 studies was ESCC $(N=1872$ patients, $90.39 \%)$. Only 189 patients $(9.13 \%)$ were diagnosed with adenocarcinoma, and $0.48 \%$ of the patients were diagnosed with cancer of other histological types. Overall, 712 (34\%) patients had stage I disease. Most of the studies [14-16, 19-26] were performed in East Asia, including Korea, Japan, and China, while 2 studies $[17,18]$ were performed in Western countries.

The radiotherapy dose, scheduling, and different chemotherapy regimens are presented in Table 1. All radiation treatments delivered in each study were definitive doses, and total doses ranged from 50 to 71.4 Gy. A platinum-based chemotherapy protocol was administered in most studies [14, 16, 17, 19-26]. The overall R0 resection rate, which was reported in 10 studies [14, 18$26]$, ranged from 83 to $100 \%$.

\section{Effect of dCRT and surgery on OS}

Figure 2 shows pooled estimates for OS in the randomised and non-randomised studies that compared dCRT with surgery. One study [16] was ineligible for the analysis of OS as only the PFS was reported. Both the short-term and long-term OS of patients treated with dCRT versus surgery were not significantly different. The pooled ORs for the 2-year and 5-year OS were 1.199 (95\% CI $0.922-1.560 ; P=0.177)$ and 0.947 (95\% CI $0.628-1.429 ; P=0.796)$, respectively. 


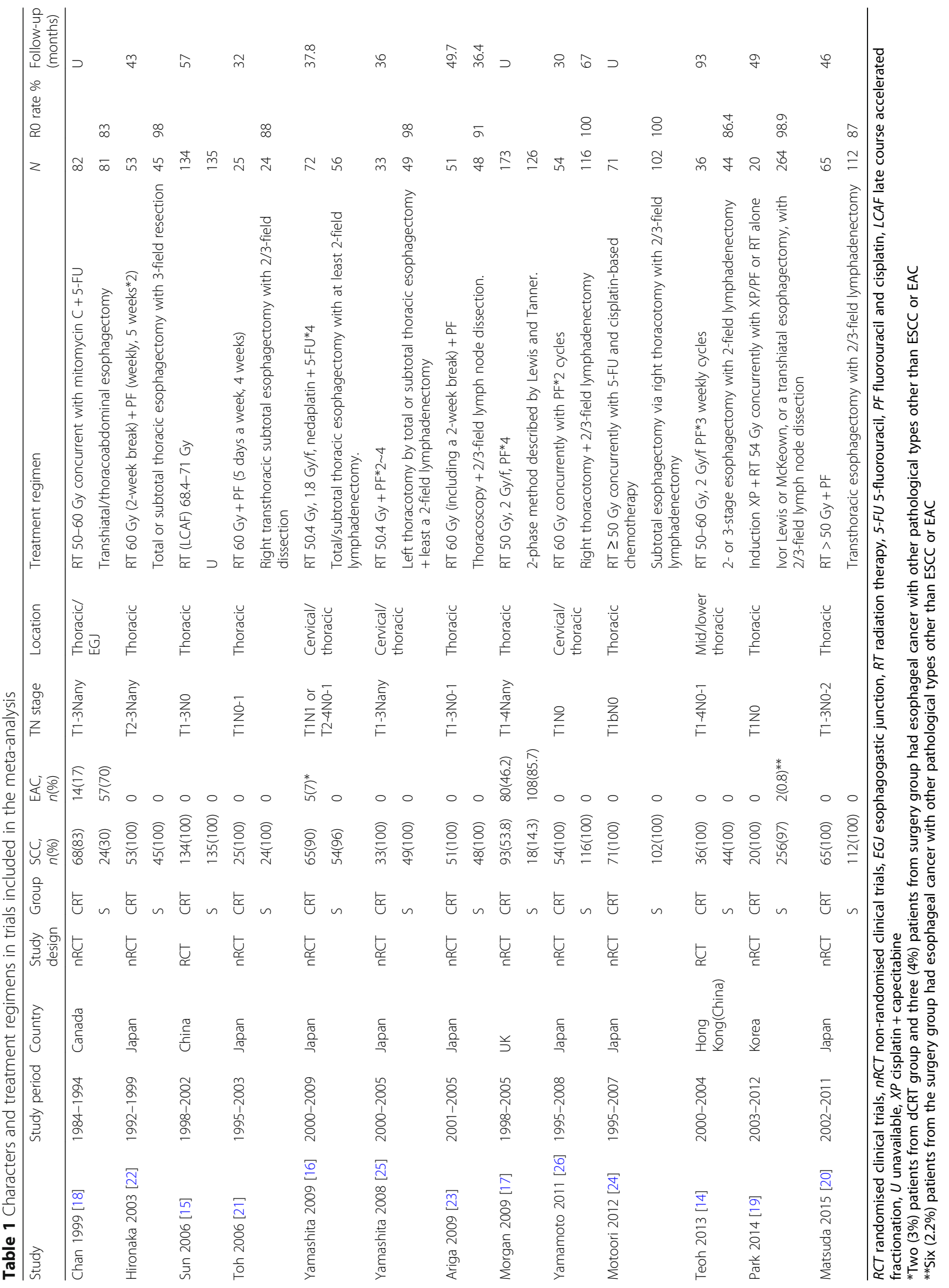




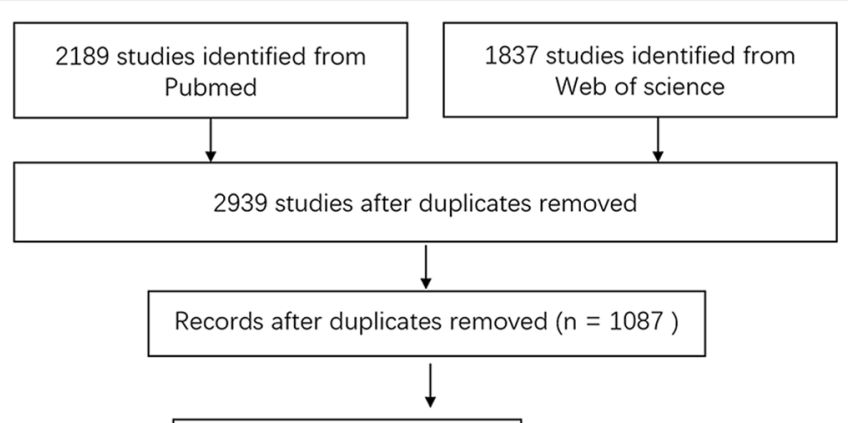

abstracts reviewed Title and abstracts screened for eligibility $(n=17)$

2 Excluded

1(Blackshaw, 2009): full text not available

1 (Carstens, 2007): no available data for patient number for each group

full-text articles assessed for eligibility $\quad(n=15)$

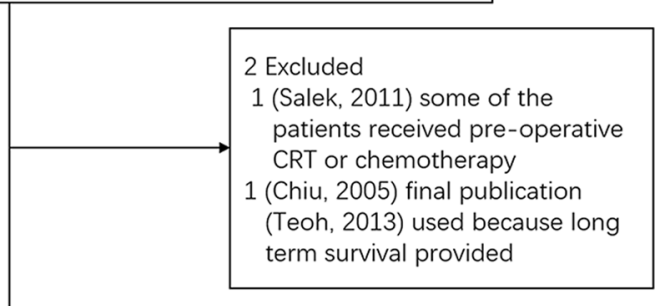

Studies included in quantitative synthesis (meta-analysis) $(n=13)$

Fig. 1 Flow diagram for article selection

A

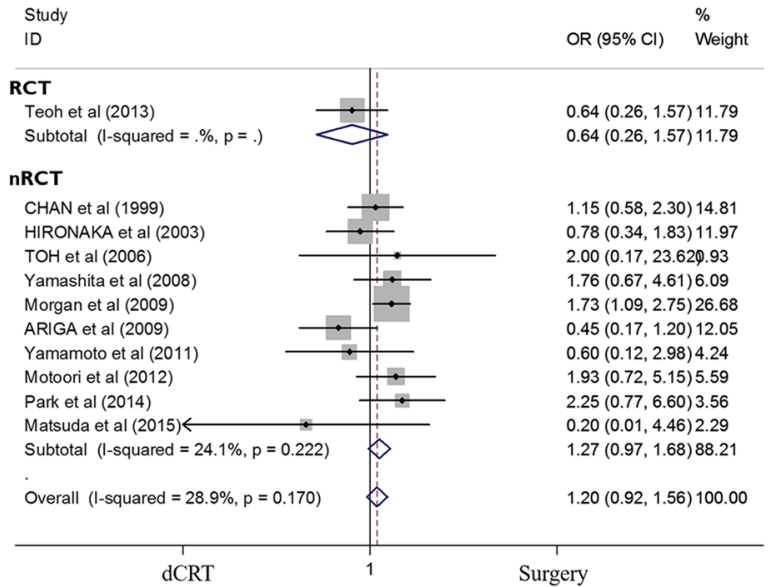

B

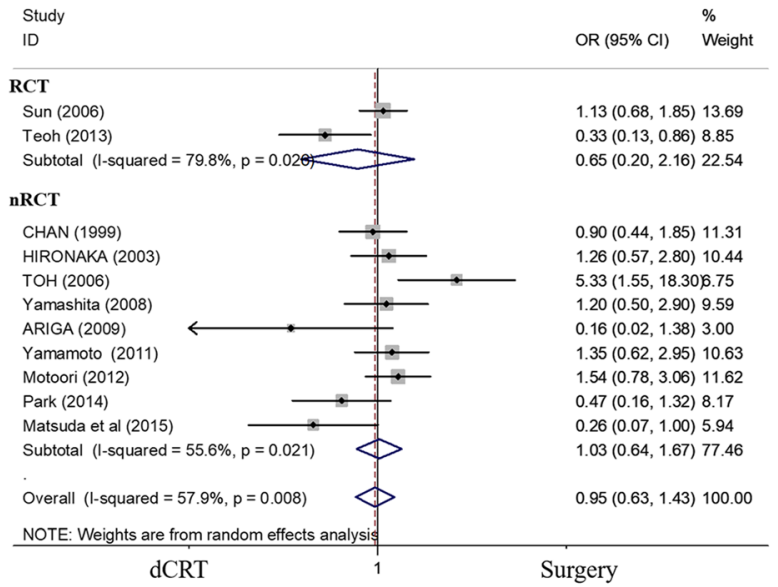

Fig. 2 Forest plot comparison of the ORs of the OS between the dCRT and surgery arms. a The OR of the 2-year OS was 1.199 (95\% CI 0.922-1.560; $P=0.177$ ). Publication bias test: $P=0.640$ (Begg's test); $P=0.240$ (Egger's test). Weights are from fixed-effects analyses. $\mathbf{b}$ The OR of the 5-year OS was 0.947 (95\% Cl 0.628-1.429; $P=0.796$ ). Publication bias test: $P=0.161$ (Begg's test), $P=0.236$ (Egger's test). Weights are from random-effects analyses 


\section{Effect of dCRT and surgery on the OS of patients with ESCC}

Nine studies $[14,15,18,20-26]$ were restricted to patients with ESCC. The pooled OR for the 5-year OS was not significantly different in patients with ESCC who were treated with dCRT compared with those who were treated with surgery $(\mathrm{OR}=1.015,95 \%$ CI 0.623-1.652; $P=0.954$ ) (Fig. 3).

\section{Subgroup analyses of the effects of dCRT and surgery in patients with different stages of esophageal cancer}

Subgroup analyses of patients with stage I and stage IIIII disease were performed, and none of the results demonstrated a significant difference between dCRT and surgery. The ORs for the 2-year OS of patients with stage I and stage II-III disease were 1.397 (95\% CI 0.740-2.638; $P=0.303$ ) and 0.418 (95\% CI 0.022-7.833; $P=0.560)$, respectively (Fig. 4). An analysis of patients with stage I ESCC was also performed, and the OR of the 2-year OS was $1.021 \quad(95 \% \quad C I \quad 0.488-2.134 ; \quad P=0.957)$ (Additional file 1: Figure S1).

\section{Subgroup analyses of patients with and without lymph node metastasis}

We identified two studies [14, 22] that included data from patients with and without positive lymph nodes. In these studies, all enrolled patients were diagnosed with ESCC. A trend towards improved survival was observed in patients with positive lymph nodes who were treated with dCRT; however, the difference was not statistically significant $(\mathrm{OR}=0.226$, 95\% CI 0.044-1.169; $P=0.076)$. For patients without lymph node metastasis, no significant difference was observed between the dCRT and surgery groups $(\mathrm{OR}=1.419,95 \%$ CI $0.613-3.289 ; P$ $=0.414$ ) (Fig. 5). However, due to the small number of studies, heterogeneity was observed among patients with lymph node metastasis between the trials due to the small number of studies.

\section{Subgroup analyses of patients from Asian and Western countries}

We performed subgroup analyses to examine OS according to different regions. In this analysis, all patients from Asian countries were diagnosed with ESCC [14, 19-26], while Western studies included patients with esophageal adenocarcinoma (EAC) (56\%) [17, 18]. The pooled results revealed no differences in terms of the 2-year OS of Asian patients who received dCRT compared with those who underwent surgery, while the estimated OR favoured surgery for patients from North America. The ORs for dCRT compared with that of surgery regarding the 2-year OS were 0.970 (95\% CI $0.674-$ 1.395; $P=0.868)$ and 1.522 (95\% CI 1.035-2.238; $P=$ 0.033) in Asian and Western patients, respectively (Fig. 6). We also performed an analysis on Asian patients with ESCC. The OR of the 2-year OS was 0.886 (95\% CI $0.604-1.302 ; P=0.538$ ) (Additional file 2: Figure $\mathrm{S} 2$ ).

\section{Effect of dCRT and surgery on PFS}

Six studies [14, 15, 18, 23-25] reported the 5-year PFS. The results showed that dCRT is equivalent to surgery in terms of the 5-year PFS (OR $=1.06,95 \%$ CI 0.79-1.42; $P=0.70$ ) (Fig. 7). The 5-year PFS for ESCC patients between the dCRT and surgery arms was not significantly

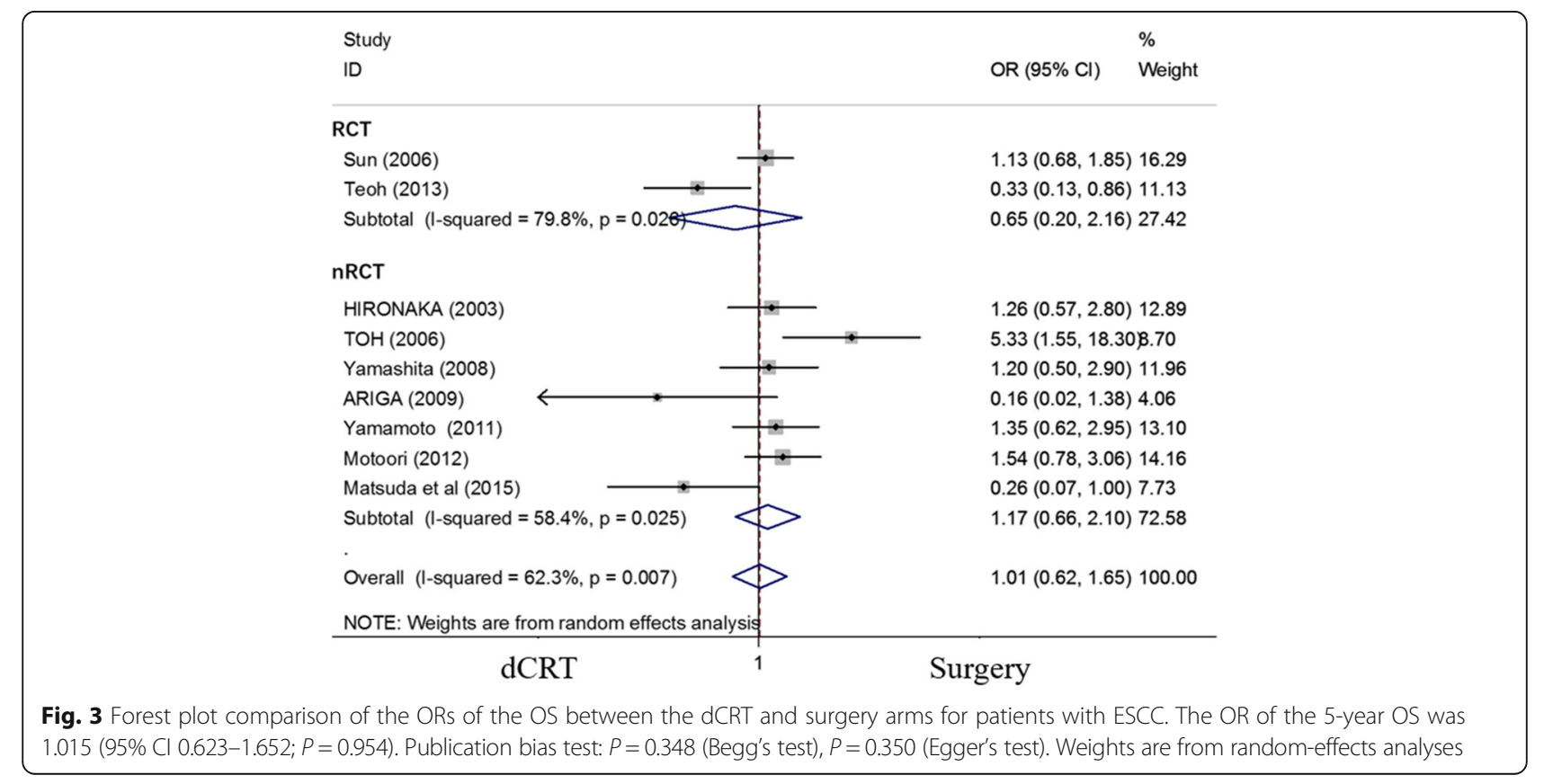




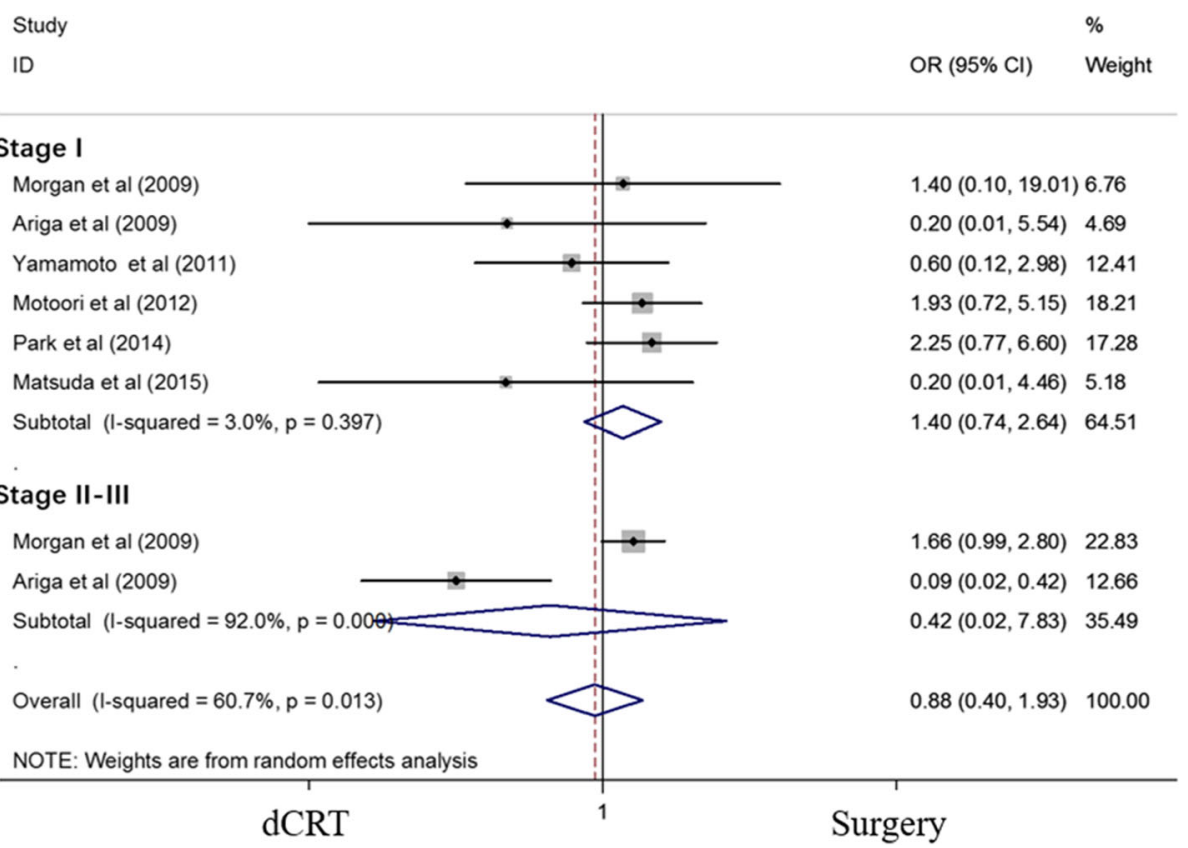

Fig. 4 Forest plot comparison of the ORs of the OS between the dCRT and surgery arms for patients with different stages of esophageal cancer. The OR of the 2-year OS for stage I esophageal cancer was 1.397 (95\% Cl $0.740-2.638 ; P=0.303$ ). Publication bias test: $P=0.133$ (Begg's test), $P=0.039$ (Egger's test). The OR of the 2-year OS for stage II-III esophageal cancer was 0.418 ( $95 \% \mathrm{Cl} 0.022-7.833 ; P=0.560)$. Publication bias (not available due to lack of studies). Weights are from random-effects analyses

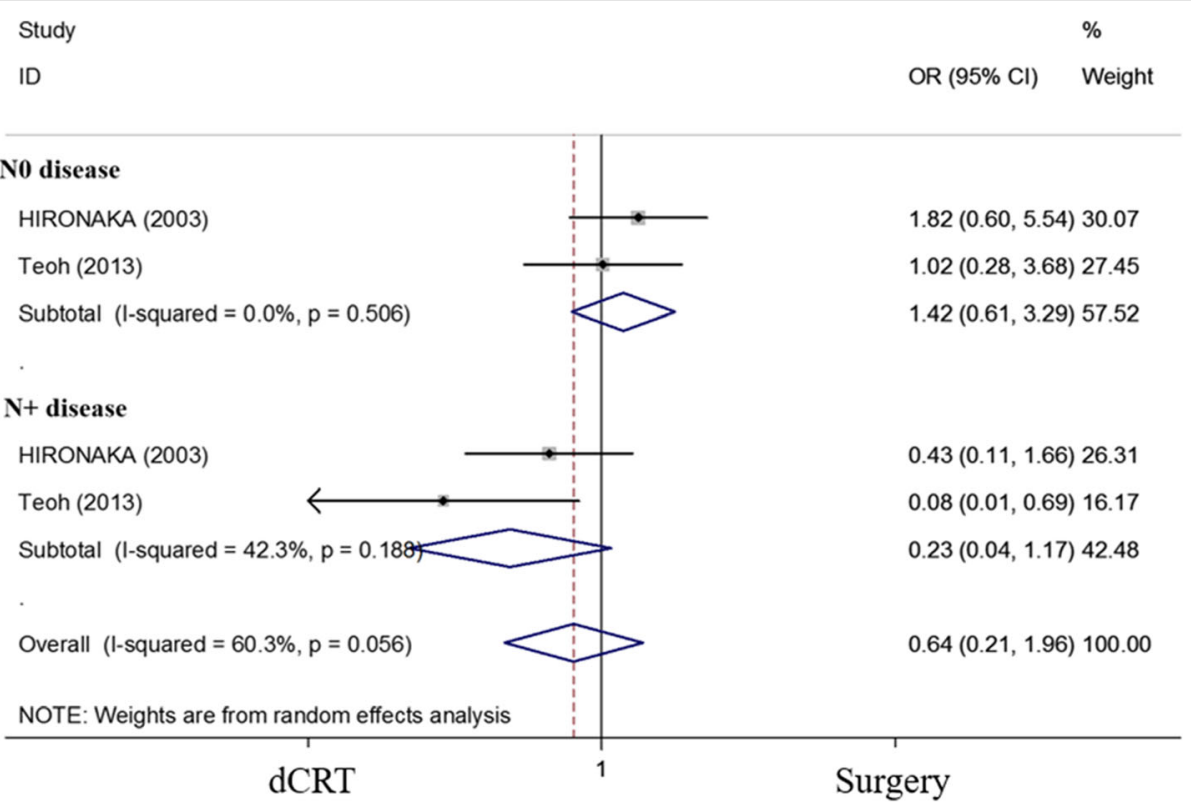

Fig. 5 Forest plot comparison of the ORs of the OS between the dCRT and surgery arms for patients with NO disease and N+ diseases. The OR of the 5-year OS for NO disease was $1.419(95 \% \mathrm{Cl} 0.613-3.289 ; P=0.414)$. Publication bias: not available due to lack of studies. The OR of the 5 -year OS for $\mathrm{N}+$ disease was $0.226(95 \% \mathrm{Cl} 0.044-1.169 ; P=0.076)$. Publication bias: not available due to lack of studies. Weights are from random-effects analyses 


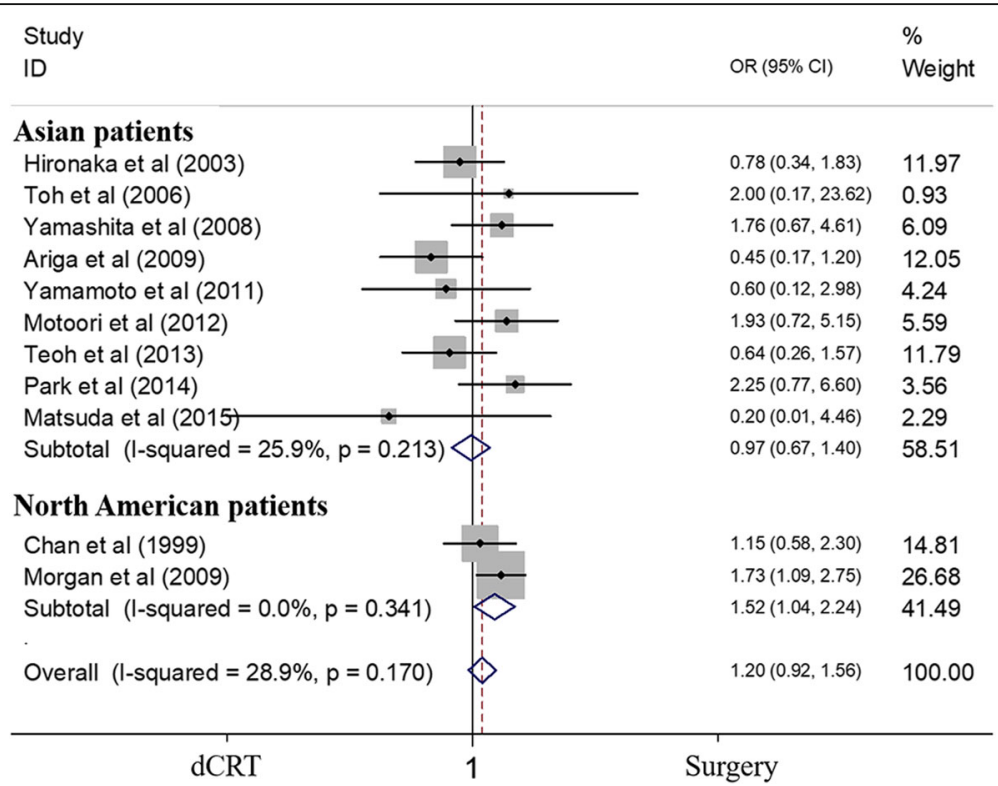

Fig. 6 Forest plot comparison of ORs of the OS between the dCRT and surgery arms for Asian patients and Western patients. The OR of the 2year OS for Asian patients was 0.970 (95\% Cl 0.674-1.395; $P=0.868$ ). Publication bias test: $P=0.835$ (Begg's test); $P=0.807$ (Egger's test). The OR of the 2-year OS for Western patients was $1.522(95 \% \mathrm{Cl} 1.035-2.238 ; P=0.033)$. Publication bias: not available due to lack of studies. Weights are from fixed-effects analyses

different. The OR of the 5-year PFS was 1.047 (95\% CI $0.623-1.760 ; P=0.862$ ) (Additional file 3: Figure S3).

\section{Discussion}

In this meta-analysis, the outcomes between dCRT and surgery as initial treatments for resectable esophageal cancer across 13 RCTs and nRCTs were compared. No statistically significant differences were observed in either short- or long-term OS or PFS. Subgroup analyses showed a trend towards improved outcomes for patients with positive lymph nodes who were treated with dCRT; however, the difference was not statistically significant. Patients from Western countries who underwent surgery had a better 2-year OS than those who received dCRT.

The number of clinical stage I esophageal cancer patients has recently increased $[27,28]$. The survival rate following surgery for submucosal tumours is high; however, the postoperative quality of life is often

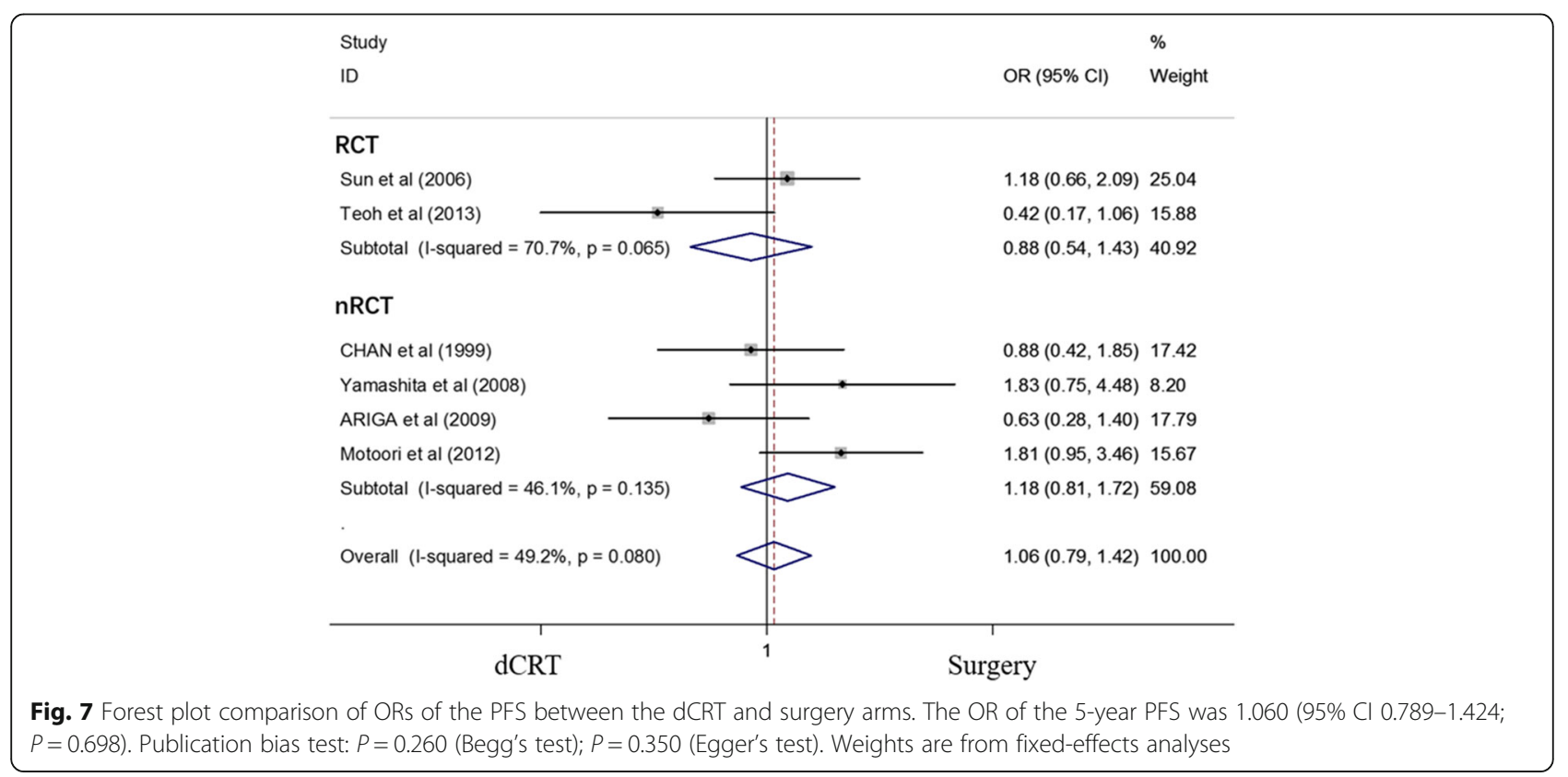


compromised. Some studies $[29,30]$ have demonstrated encouraging clinical results for dCRT in these patients. In this meta-analysis, the 2-year OS of patients with stage I esophageal cancer was comparable between the $\mathrm{dCRT}$ and esophagostomy groups. Therefore, dCRT may be considered a treatment modality in selected patients. The ongoing JCOG0502 study by the Japan Clinical Oncology Group is investigating the non-inferiority of dCRT compared with surgery for stage I esophageal cancer patients.

Esophageal cancer is characterised by a high rate of lymph node metastasis [31], which is the most reliable predictor of survival after surgery [32]. In addition, because its pattern of spread is not always predictable and since skip node metastases may also occur, lymph node dissections may be difficult to perform. As suggested by our subgroup analyses, dCRT was superior to surgery among patients with lymph node metastases.

The pathological types of esophageal cancer are characterised by obvious demographic variations. The incidence of ESCC is much higher in Asia than in Western countries, whereas EAC accounts for only $1-4 \%$ of cases in Asian countries [2]. In addition, the incidence of EAC in Western countries is increasing rapidly [33]. We extracted data from all patients with ESCC and found no difference between dCRT and surgery in terms of long-term OS. Moreover, the subgroup analysis of the geographic areas showed that the 2-year OS was comparable between Asian patients who received dCRT and those who received surgery. In Western patients, surgical treatment has obvious therapeutic benefits. Studies on preoperative chemoradiotherapy $[4,6,34]$ have shown that the pathological complete response rate of patients with EAC was lower than that of patients with ESCC. In this meta-analysis, two studies enrolled patients with EAC from Western countries $[17,18]$ (proportion of EAC, $44.1 \%$ and $62.9 \%$ ), whereas almost all patients from Asian countries had ESCC. In addition, these two studies, which were performed in Western countries, included a large proportion of patients with lower esophageal cancer (66.9\% and $77.3 \%)$. Patients with lower esophageal cancer were more amenable to surgery.

The progression rate of esophageal cancer is usually high when treated with either dCRT or surgery alone [34-37]. For long-term PFS, dCRT is equivalent to surgery when used as the initial treatment modality. A multidisciplinary approach is the ideal strategy, especially for the treatment of esophageal cancer.

This meta-analysis has several limitations. First, retrospective studies were included; therefore, selection bias may exist. For example, patients treated with dCRT in these studies were diagnosed with more advanced disease than those treated with surgery. Second, individual results from each patient were not applied. Third, modest heterogeneity was observed in terms of the surgical methods that were used and the dosing schedules between studies. In addition, the number of studies in the subgroup analyses was limited, especially those that included patients with lymph node metastasis and those with Western ethnicity. Finally, the studies were limited to two languages, which may present another bias.

\section{Conclusions}

Our study demonstrates that dCRT is similar to surgery as an initial treatment for esophageal cancer with respect to the long-term survival of patients. Surgery may lead to a better OS in patients from Western countries, but further randomised trials are required to confirm these results.

\section{Additional files}

Additional file 1: Figure S1. Forest plot comparison of ORs of the OS between the dCRT and surgery arms for stage I ESCC patients. The OR of the 2-year OS was 1.021 (95\% Cl 0.488-2.134; $P=0.957)$. Publication bias test: $P=0.308$ (Begg's test); $P=0.042$ (Egger's test). Weights are from fixed-effects analyses. (TIF $450 \mathrm{~kb}$ )

Additional file 2: Figure S2. Forest plot comparison of ORs of the OS between the dCRT and surgery arms for Asian ESCC patients. The OR of the 2-year OS was 0.886 (95\% Cl 0.604-1.302; $P=0.538)$. Publication bias test: $P=0.902$ (Begg's test); $P=0.769$ (Egger's test). Weights are from fixed-effects analyses. (TIF $764 \mathrm{~kb}$ )

Additional file 3: Figure S3. Forest plot comparison of ORs of the PFS between the dCRT and surgery arms for ESCC patients. The OR of the 5-year PFS was 1.047 (95\% Cl 0.623-1.760; $P=0.862$ ). Publication bias test: $P=0.462$ (Begg's test); $P=0.432$ (Egger's test). Weights are from randomeffects analyses. (TIF $601 \mathrm{~kb}$ )

\section{Abbreviations}

5-FU: 5-Fluorouracil; Cl: Confidence interval; dCRT: Definitive chemoradiotherapy; EAC: Esophageal adenocarcinoma; ESCC: Esophageal squamous cell carcinoma; LCAF: Late course accelerated fractionation; nRCT: Non-randomised clinical trial; OR: Odds ratio; OS: Overall survival; PF: Fluorouracil and cisplatin; PFS: Progression-free survival; PRISMA: Preferred Reporting Items for Reviews and Meta-Analyses; RCT: Randomised clinical trial; RT: Radiation therapy; U: Unavailable; XP: Cisplatin + capecitabine

\section{Availability of data and materials}

The datasets generated and/or analysed during the current study are available on PubMed (http://www.ncbi.nlm.nih.gov/pubmed) and Web of Science (http://www.isiknowledge.com/). The search terms used were as follows: (esophageal cancer or esophageal neoplasms) and (chemoradiotherapy or chemoradiation therapy) and (esophagectomy OR surgery).

\section{Authors' contributions}

MWM designed the study, analysed the data, and drafted the manuscript. $X B G, M C, M X$, and $L L$ collected and verified the data. $H Y, M Z$, and $L L$ analysed and interpreted the data. LQC and XSG designed the study, interpreted the data, revised the manuscript, and made the decision to submit for publication. All authors read and approved the final manuscript.

Ethics approval and consent to participate Not applicable.

Consent for publication

Not applicable. 


\section{Competing interests}

The authors declare that they have no competing interests.

\section{Publisher's Note}

Springer Nature remains neutral with regard to jurisdictional claims in published maps and institutional affiliations.

\section{Author details}

'Department of Radiation Oncology, Peking University First Hospital, No.7 Xishiku Street, Beijing 100034, People's Republic of China. ${ }^{2}$ Department of Medical and Pharmaceutical Science and Technology Strategy Research, Institute of Medical Information, Chinese Academy of Medical Sciences, No. 3 Yabao Road, Beijing, China. ${ }^{3}$ Department of Thoracic Surgery, West China School of Medicine/West China Hospital of Sichuan University, No. 37 Guoxue Alley, Chengdu 610041, Sichuan, People's Republic of China.

\section{Received: 13 May 2018 Accepted: 3 August 2018}

\section{Published online: 17 August 2018}

\section{References}

1. Mariette C, Piessen G, Triboulet JP. Therapeutic strategies in oesophageal carcinoma: role of surgery and other modalities. Lancet Oncol. 2007;8:545-53.

2. Hongo M, Nagasaki Y, Shoji T. Epidemiology of esophageal cancer: Orient to Occident. Effects of chronology, geography and ethnicity. J Gastroenterol Hepatol. 2009:24:729-35.

3. Sjoquist KM, Burmeister BH, Smithers BM, Zalcberg JR, Simes RJ, Barbour A Gebski V. Survival after neoadjuvant chemotherapy or chemoradiotherapy for resectable oesophageal carcinoma: an updated meta-analysis. Lancet Oncol. 2011;12:681-92.

4. van Hagen P, Hulshof MC, van Lanschot JJ, Steyerberg EW, van Berge Henegouwen MI, Wijnhoven BP, Richel DJ, Nieuwenhuijzen GA, Hospers GA, Bonenkamp JJ, et al. Preoperative chemoradiotherapy for esophageal or junctional cancer. N Engl J Med. 2012;366:2074-84

5. Bosset JF, Gignoux M, Triboulet JP, Tiret E, Mantion G, Elias D, Lozach P, Ollier JC, Pavy JJ, Mercier M, Sahmoud T. Chemoradiotherapy followed by surgery compared with surgery alone in squamous-cell cancer of the esophagus. N Engl J Med. 1997;337:161-7.

6. Urba SG, Orringer MB, Turrisi A, lannettoni M, Forastiere A, Strawderman M. Randomized trial of preoperative chemoradiation versus surgery alone in patients with locoregional esophageal carcinoma. J Clin Oncol. 2001;19:305-13.

7. Nygaard K, Hagen S, Hansen HS, Hatlevoll R, Hultborn R, Jakobsen A, Mantyla M, Modig H, Munck-Wikland E, Rosengren B, et al. Pre-operative radiotherapy prolongs survival in operable esophageal carcinoma: a randomized, multicenter study of pre-operative radiotherapy and chemotherapy. The second Scandinavian trial in esophageal cancer. World J Surg. 1992;16:1104-9. discussion 1110

8. Walsh TN, Noonan N, Hollywood D, Kelly A, Keeling N, Hennessy TP. A comparison of multimodal therapy and surgery for esophageal adenocarcinoma. N Engl J Med. 1996:335:462-7.

9. Birkmeyer JD, Stukel TA, Siewers AE, Goodney PP, Wennberg DE, Lucas FL. Surgeon volume and operative mortality in the United States. N Engl J Med. 2003;349:2117-27.

10. Steyerberg EW, Neville BA, Koppert LB, Lemmens VE, Tilanus HW, Coebergh JW, Weeks JC, Earle CC. Surgical mortality in patients with esophageal cancer: development and validation of a simple risk score. J Clin Oncol. 2006:24:4277-84

11. D'Journo XB, Thomas PA. Current management of esophageal cancer. J Thorac Dis. 2014:6(Suppl 2):S253-64.

12. Kato H, Sato A, Fukuda H, Kagami Y, Udagawa H, Togo A, Ando N, Tanaka O, Shinoda M, Yamana H, Ishikura S. A phase II trial of chemoradiotherapy for stage I esophageal squamous cell carcinoma: Japan Clinical Oncology Group Study (JCOG9708). Jpn J Clin Oncol. 2009;39:638-43.

13. Liberati A, Altman DG, Tetzlaff J, Mulrow C, Gotzsche PC, loannidis JP, Clarke M, Devereaux PJ, Kleijnen J, Moher D. The PRISMA statement for reporting systematic reviews and meta-analyses of studies that evaluate healthcare interventions: explanation and elaboration. BMJ. 2009;339:b2700.

14. Teoh AY, Chiu PW, Yeung WK, Liu SY, Wong SK, Ng EK. Long-term survival outcomes after definitive chemoradiation versus surgery in patients with resectable squamous carcinoma of the esophagus: results from a randomized controlled trial. Ann Oncol. 2013;24:165-71.
15. Sun XD, Yu JM, Fan XL, Ren RM, Li MH, Zhang GL. Randomized clinical study of surgery versus radiotherapy alone in the treatment of resectable esophageal cancer in the chest. Zhonghua Zhong Liu Za Zhi. 2006;28: 784-7.

16. Yamashita H, Okuma K, Seto Y, Mori K, Kobayashi S, Wakui R, Ohtomo K, Nakagawa K. A retrospective comparison of clinical outcomes and quality of life measures between definitive chemoradiation alone and radical surgery for clinical stage II-III esophageal carcinoma. J Surg Oncol. 2009;100:435-41.

17. Morgan MA, Lewis WG, Casbard A, Roberts SA, Adams R, Clark GW, Havard TJ, Crosby TD. Stage-for-stage comparison of definitive chemoradiotherapy, surgery alone and neoadjuvant chemotherapy for oesophageal carcinoma. Br J Surg. 2009;96:1300-7.

18. Chan A, Wong A. Is combined chemotherapy and radiation therapy equally effective as surgical resection in localized esophageal carcinoma? Int J Radiat Oncol Biol Phys. 1999:45:265-70.

19. Park I, Kim YH, Yoon DH, Park SR, Kim HR, Kim JH, Jung HY, Lee GH, Cho KJ, Kim SB. Non-surgical treatment versus radical esophagectomy for clinical T1N0M0 esophageal carcinoma: a single-center experience. Cancer Chemother Pharmacol. 2014:74:995-1003.

20. Matsuda S, Tsubosa Y, Niihara M, Sato H, Takebayashi K, Kawamorita K, Mori K, Tsushima T, Yokota T, Ogawa H, et al. Comparison of transthoracic esophagectomy with definitive chemoradiotherapy as initial treatment for patients with esophageal squamous cell carcinoma who could tolerate transthoracic esophagectomy. Ann Surg Oncol. 2015;22:1866-73.

21. Toh Y, Ohga T, Itoh S, Kabashima A, Yamamoto K, Adachi E, Sakaguchi Y, Okamura T, Hirata $\mathrm{H}$. Treatment results of radical surgery and definitive chemoradiotherapy for patients with submucosal esophageal squamous cell cancinomas. Anticancer Res. 2006:26:2487-91.

22. Hironaka S, Ohtsu A, Boku N, Muto M, Nagashima F, Saito H, Yoshida S, Nishimura M, Haruno M, Ishikura S, et al. Nonrandomized comparison between definitive chemoradiotherapy and radical surgery in patients with $\mathrm{T}(2-3) \mathrm{N}$ (any) M(0) squamous cell carcinoma of the esophagus. Int J Radiat Oncol Biol Phys. 2003;57:425-33.

23. Ariga H, Nemoto K, Miyazaki S, Yoshioka T, Ogawa Y, Sakayauchi T, Jingu K, Miyata G, Onodera K, Ichikawa $\mathrm{H}$, et al. Prospective comparison of surgery alone and chemoradiotherapy with selective surgery in resectable squamous cell carcinoma of the esophagus. Int J Radiat Oncol Biol Phys. 2009:75:348-56.

24. Motoori M, Yano M, Ishihara R, Yamamoto S, Kawaguchi Y, Tanaka K, Kishi K, Miyashiro I, Fujiwara $Y$, Shingai T, et al. Comparison between radical esophagectomy and definitive chemoradiotherapy in patients with clinical T1bN0M0 esophageal cancer. Ann Surg Oncol. 2012;19:2135-41.

25. Yamashita H, Nakagawa K, Yamada K, Kaminishi M, Mafune K, Ohtomo K. A single institutional non-randomized retrospective comparison between definitive chemoradiotherapy and radical surgery in 82 Japanese patients with resectable esophageal squamous cell carcinoma. Dis Esophagus. 2008;21:430-6.

26. Yamamoto $S$, Ishihara $R$, Motoori M, Kawaguchi $Y$, Uedo N, Takeuchi $Y$, Higashino K, Yano M, Nakamura S, lishi H. Comparison between definitive chemoradiotherapy and esophagectomy in patients with clinical stage esophageal squamous cell carcinoma. Am J Gastroenterol. 2011;106: 1048-54.

27. Ozawa S. Comprehensive registry of esophageal cancer in Japan. In: bits 3rd annual world cancer congress-2012; 2012. p. 21-47.

28. Kodaira T, Fuwa N, Tachibana H, Nakamura T, Tomita N, Nakahara R, Inokuchi $H$, Mizoguchi N, Takada A. Retrospective analysis of definitive radiotherapy for patients with superficial esophageal carcinoma: consideration of the optimal treatment method with a focus on late morbidity. Radiother Oncol. 2010:95:234-9.

29. Yamada K, Murakami M, Okamoto Y, Okuno Y, Nakajima T, Kusumi F, Takakuwa H, Matsusue S. Treatment results of chemoradiotherapy for clinical stage I (T1NOM0) esophageal carcinoma. Int J Radiat Oncol Biol Phys. 2006:64:1106-11.

30. Nemoto K, Yamada S, Hareyama M, Nagakura H, Hirokawa Y. Radiation therapy for superficial esophageal cancer: a comparison of radiotherapy methods. Int J Radiat Oncol Biol Phys. 2001;50:639-44.

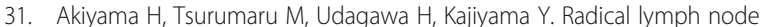
dissection for cancer of the thoracic esophagus. Ann Surg. 1994;220:364-72. discussion 372-363

32. Zhu YM, Li MH, Kong L, Yu JM. Postoperative radiation in esophageal squamous cell carcinoma and target volume delineation. Oncotargets Therapy. 2016;9:4187-96. 
33. Bollschweiler E, Wolfgarten E, Gutschow C, Holscher AH. Demographic variations in the rising incidence of esophageal adenocarcinoma in white males. Cancer. 2001;92:549-55.

34. Burmeister BH, Smithers BM, Gebski V, Fitzgerald L, Simes RJ, Devitt $P$, Ackland S, Gotley DC, Joseph D, Millar J, et al. Surgery alone versus chemoradiotherapy followed by surgery for resectable cancer of the oesophagus: a randomised controlled phase III trial. Lancet Oncol. 2005;6: 659-68.

35. Stahl M, Stuschke M, Lehmann N, Meyer HJ, Walz MK, Seeber S, Klump B, Budach W, Teichmann R, Schmitt M, et al. Chemoradiation with and without surgery in patients with locally advanced squamous cell carcinoma of the esophagus. J Clin Oncol. 2005;23:2310-7.

36. Minsky BD, Pajak TF, Ginsberg RJ, Pisansky TM, Martenson J, Komaki R, Okawara G, Rosenthal SA, Kelsen DP. INT 0123 (Radiation Therapy Oncology Group 94-05) phase III trial of combined-modality therapy for esophageal cancer: high-dose versus standard-dose radiation therapy. J Clin Oncol. 2002:20:1167-74

37. Bedenne L, Michel P, Bouche O, Milan C, Mariette C, Conroy T, Pezet D, Roullet B, Seitz JF, Herr JP, et al. Chemoradiation followed by surgery compared with chemoradiation alone in squamous cancer of the esophagus: FFCD 9102. J Clin Oncol. 2007;25:1160-8.

Ready to submit your research? Choose BMC and benefit from:

- fast, convenient online submission

- thorough peer review by experienced researchers in your field

- rapid publication on acceptance

- support for research data, including large and complex data types

- gold Open Access which fosters wider collaboration and increased citations

- maximum visibility for your research: over $100 \mathrm{M}$ website views per year

At $\mathrm{BMC}$, research is always in progress.

Learn more biomedcentral.com/submissions 